\title{
Aportaciones para la educación psicomotriz, aprendizajes lectoescritores y la asimilación del esquema corporal en niños/as de 5 años
}

\author{
Ana Aguilar Anisa, Fátima Llamas Salguero y Verónica López Fernández. Universidad \\ Internacional de la Rioja
}

\author{
Recepción: 18 de agosto de 2015 | Revisión: 21 de agosto de 2015 | Aceptación/Publicación: 4 de septiembre de 2015 \\ Correspondencia: fatima.IImas@unir.net \\ Citar: Aguilar, A., Llamas-Salguero, F. y Lopez-Fernandez, V. (2015). Aportaciones para la educación psicomotriz, aprendizajes \\ lectoescritores y la asimilación del esquema corporal en niños/as de 5 años. ReiDoCrea, 4, $219-227$. \\ http://hdl.handle.net/10481/37248
}

Resumen: El presente trabajo pone énfasis en destacar la importancia que posee la psicomotricidad en el alumnado de Educación Infantil. Se pretende concienciar tanto al ámbito escolar como al familiar sobre la relación entre psicomotricidad y el aprendizaje del alumnado. Para ello se analiza una serie de variables como los patrones básicos de movimiento, la lateralidad y los procesos lectoescritores. Los patrones básicos de movimiento se miden a través de la prueba de Santiuste, Martín Lobo y Ayala, (2005) que valora arrastre, gateo, marcha, triscado, carrera, tono muscular y control postural. Con la dominancia lateral se obtiene información del lado dominante en visión, audición, lateralidad manual y pédica, evaluado con la prueba neuropsicológica del Instituto de Neuropsicología y Educación, adaptado por Martín Lobo, García Castellón, Rodríguez y Vallejo (2011) y los procesos lectoescritores mediante un dictado de vocales, consonantes y números. Estas pruebas han sido aplicadas a una muestra de 30 alumnos de 5 años de edad del último curso de Educación Infantil. Los resultados nos indican que existe correlación entre un adecuado desarrollo psicomotor y un correcto aprendizaje de procesos lectoescritores.

Palabras Clave: Lectura y Escritura | Lateralidad cerebral

Contributions for Psychomotor Education, Learning of Reading and Writing Processes, and Assimilation of Body Scheme in 5-year-old Children

Abstract: This paper emphasizes on the importance that has the motor skills in students of Primary Education. It aims to raise the awareness of the school environment and the family about the relationship between motor skills and student learning. To this end a number of variables is analyzed as the basic movement patterns, laterality and reading and writing processes. The basic movement patterns are measured through test Santiuste, Martin Lobo and Ayala (2005) that values creeping, crawling, running, unset, race, muscle tone and postural control. With lateral dominance information is obtained from the dominant side in vision, hearing, and PEDIS handedness, evaluated neuropsychological test Neuropsychology and Education Institute, adapted by Martin Lobo, Garcia Castellon, Rodriguez, and Vallejo (2011) and reading and writing processes through a dictate of vowels, consonants and numbers. These tests have been applied to a sample of 30 students from 5 years old the last year of kindergarten. The results indicate that there is a correlation between adequate psychomotor development and learning proper processes reading and writing.

Key Word: Reading and Writing | Lateral Dominance

\section{Introducción}

El desarrollo del niño en la etapa de Educación Infantil es fundamental. Durante este periodo se conforman aspectos relevantes para su desarrollo integral como la toma de conciencia de sí mismo, de su entorno, madurez intelectual y afectiva (Aucouturier, Lapierre, 2000).

Tanto el contexto escolar como contexto familiar juegan un papel fundamental en esta etapa. Por tanto, educadores y padres deberán prestar al niño toda la ayuda posible para que éste proceda a asimilar e integrar todas las vivencias que le proporcionará este periodo.

Un ambiente basado en la estimulación va a provocar que el niño se vea inmerso y sea partícipe de un enriquecedor proceso de enseñanza-aprendizaje.

La importancia que adquiere la motricidad global en el proceso de aprendizaje del alumnado hace necesario que desde las instituciones educativas se contemple en el 
currículo programaciones destinadas a conseguir tanto un desarrollo psicomotor como motor idóneos y acordes con su rango de edad.

Por tanto, hemos de incidir en que adquieran un aprendizaje basado en unos objetivos, contenidos, metodología y evaluación de calidad, ya que en los primeros años de escolarización se establecerán las bases fundamentales de aprendizajes como pueden ser la lectura y la escritura.

En muchas ocasiones el proceso de enseñanza-aprendizaje se lleva a cabo correctamente pero no se realizan las evaluaciones pertinentes para determinar posibles dificultades.

En este trabajo se van a valorar aspectos de la motricidad como el arrastre, gateo, marcha, triscado, carrera, tono muscular y control postural en alumnos, la lateralidad, y se va a comprobar si existe una relación significativa con los aprendizajes lectoescritores.

Una definición que recoge todas las acepciones del término la han elaborado De Lièvre y Staes (1992): la psicomotricidad desde un enfoque globalizado conjuga dos aspectos fundamentales como psicología y movimiento o como hacen referencia numerosos autores psiquismo y motricidad. Por tanto, el concepto no se puede contemplar desde un punto de vista aislado, sino que deben darse ambos para que exista una interacción del niño con el mundo que le rodea.

Según Martín Lobo (2003) existen factores que influyen en el aprendizaje, pero en el que recae la mayor relevancia es la motricidad. Alude a la implicación de las áreas motrices en la lectura y de forma más directa en la escritura.

Henrietta Leiner y Alan Leiner (1993) fueron los pioneros, a través de un estudio centrado en el cerebelo, en evidenciar un enlace entre mente y cuerpo. El cerebelo fue comparado con un ordenador capaz de hacer contribuciones tanto en la destreza motora como en destreza mental de los seres humanos, requeridos para la aparición de un fluido lenguaje. Por todo ello Strick (2009) estableció otra relación con la activación del cerebelo incluyendo tareas diseñadas para evaluar la memoria de trabajo, atención, el control ejecutivo, el lenguaje, el aprendizaje, la emoción y el dolor.

Hannaford (1995) concede importancia al sistema vestibular, encargado de la audición a través del oído interno, y al sistema cerebelar, encargado de la actividad motora, haciendo énfasis en que son los primeros en madurar. De ahí la importancia que recae en juegos que estimulan el oído interno a través de balanceo y salto.

Por todo ello un reciente estudio elaborado por Chadock- Heyman, Erickson, Holtrop, Voss, Pontifex, Rai-ne, Hillman y Kramer (2014) destaca la importancia de desarrollar una buena forma física para optimizar la salud cerebral, dejando en evidencia que los niños con peores resultados en la condición física correlacionan con una salud cerebral menos buena.

Como podemos comprobar tras este recorrido conceptual, histórico y neuropsicológico, existe bastante evidencia empírica que demuestra la relación existente entre motricidad y aprendizaje. 
Los primeros momentos del desarrollo del niño se caracterizan por la ausencia del control motor en la escritura hasta que a través de la actividad exploratoria, la estimulación y la madurez neuromotora hagan que el niño consiga regular el trazo.

Los primeros trazos los realizarán de forman impulsiva, caracterizados por el mero placer del movimiento del brazo y mano.

La lateralidad va a jugar un papel muy importante en los procesos lectoescritores, pues una lateralización tardía puede influir negativamente en dichos aprendizajes y, por derivación, en el rendimiento académico. Resulta, por tanto, fundamental conocer la organización lateral del alumnado para poder descubrir si existe algún factor influyente en la integración de ambos hemisferios.

\begin{tabular}{|l|l|l|l|l|l|}
\hline \multicolumn{4}{|c|}{ Aabla 1 } \\
\hline Lóbulo frontal & Lóbulo parietal & Lóbulo temporal & $\begin{array}{l}\text { Lóbulo } \\
\text { occipital }\end{array}$ & $\begin{array}{l}\text { Cuerpo calloso } \\
\text { Sistema } \\
\text { límbico }\end{array}$ \\
\hline $\begin{array}{l}\text { Pensamiento y } \\
\text { autocontrol }\end{array}$ & $\begin{array}{l}\text { Información táctil, } \\
\text { sensorial y } \\
\text { pensamiento }\end{array}$ & $\begin{array}{l}\text { Audición, fonética, } \\
\text { lectura, escritura y } \\
\text { ortografía }\end{array}$ & $\begin{array}{l}\text { Visión: palabras, } \\
\text { frases, líneas. }\end{array}$ & $\begin{array}{l}\text { Informaciones de } \\
\text { ambos hemisferios }\end{array}$ & $\begin{array}{l}\text { Motivación e } \\
\text { interés }\end{array}$ \\
\hline
\end{tabular}

Además, es necesario mencionar que en el proceso de lateralización influyen funciones visuales, auditivas, táctiles, sentido espacio- temporal, coordinación visual y motriz y control manual y del trazo. En concreto en el proceso lector intervendrá la convergencia binocular, ambos hemisferios cerebrales, el cuerpo calloso y las estructuras parietales. El hemisferio dominante se encargará de realizar la decodificación de la palabra por lo que actuarán las áreas occipitales (Área de Broca y Wernicke) mientras que el otro hemisferio relacionará toda la información que se ha obtenido por la vía visual y el cuerpo calloso. Las áreas parietales integrarán los aspectos analíticos del hemisferio izquierdo y los globales del hemisferio derecho. En los procesos de escritura en función de lo que se demande habrá un área en la que recaiga el peso de actuación. Para partir de una idea intervendrán las áreas frontales, para realizar un dictado las áreas temporales y para el copiado de un texto las áreas visuales y occipitales.

\section{Método}

Este estudio analiza la relación entre las variables: patrones básicos de movimiento, lateralidad, procesos lectoescritores en una muestra de 30 estudiantes de un colegio extremeño del último curso de Educación Infantil, formada por 15 niñas y 15 niños de edades comprendidas entre 5 y 6 años.

El objetivo general del presente estudio es investigar la psicomotricidad desde el plano neuropsicológico en la etapa de Educación Infantil, concretamente en alumnos de 5 años.

Los objetivos específicos son los que se detallan a continuación:

- Demostrar la importancia de la psicomotricidad en Educación Infantil.

- Demostrar la relación existente entre psicomotricidad y procesos lectoescritores.

El diseño llevado a cabo en este estudio ha sido experimental, ex-post-facto, de tipo descriptivo de observación, ya que tiene como base la investigación-acción de un profesional en el aula. 


\section{Instrumentos}

Las variables medidas en el presente estudio se valoraron a través de la observación directa. Se midieron patrones básicos de movimiento: arrastre, gateo, marcha, triscado, carrera, tono muscular y control postural; lateralidad: lado dominante en visión, audición, lateralidad manual y pédica; y escritura de vocales, algunas consonantes y números.

Los instrumentos utilizados son:

- Prueba de Patrones Básicos de Movimiento

- Prueba de lateralidad

- Prueba de escritura

La prueba de Patrones Básicos de Movimiento evalúa aspectos psicomotores (Santiuste, Martín Lobo y Ayala, 2005) a través de un registro que indica en qué medida supera el patrón en función de los siguientes criterios:

1. No supera severo

2. No supera medio

3. No supera medio

4. No supera leve

5. Supera

Para obtener una puntuación de 5 en cada caso se tiene que dar lo que a continuación se menciona en cada uno de los patrones:

- Arrastre: patrón cruzado automatizado y con soltura.

- Gateo: patrón cruzado automatizado y con soltura.

- Marcha: camina en patrón cruzado con agilidad.

- Triscado: Realiza el triscado en patrón cruzado armónicamente, con balanceo de los brazos desde los hombros y elevación de rodillas

- Carrera: corre en patrón cruzado con equilibrio y armonía, con los brazos flexionados en el codo y sin excesivo golpeo sobre el suelo.

- Tono muscular: ejerce una gran tensión o fuerza muscular.

- Control postural: Mantiene la cabeza en la línea media, hombros y caderas a la misma altura, piernas rectas y pies paralelos.

Para valorar la lateralidad se ha utilizado el test de lateralidad de la prueba neuropsicológica del Instituto de Neuropsicología y Educación, adaptado por Martín Lobo, García Castellón, Rodríguez y Vallejo (2011). Se trata de una prueba de aplicación individual que se puede aplicar a partir de los cuatro años de edad, en la que se obtiene información del lado dominante en visión, audición, lateralidad manual y pédica. Se evalúa cada una de las partes a través de la realización de diez pruebas o ejercicios en los que se anota si ha utilizado el lado izquierdo o derecho. Por lo que si usan un lado más de 6 veces se considera que la lateralidad se define de ese lado. En el caso de que haya 5 de un lado y 5 de otro se determinará que está sin definir en esa parte del cuerpo.

Para valorar los aprendizajes lectoescritores se ha llevado a cabo la aplicación de una prueba de dictado de vocales, consonantes que se han trabajado $(\mathrm{m}, \mathrm{p}, \mathrm{I}, \mathrm{t}, \mathrm{s}, \mathrm{d}) \mathrm{y}$ números del 1 al 9 , pues la evaluación del alumnado se ha realizado en el primer trimestre del último curso de Educación Infantil. 


\section{Análisis}

En cuanto a la prueba de lectoescritura se realizó un análisis global de dicha prueba donde encontramos que 12 niños/as tienen 3 ó más errores, mientras 18 niños/as tienen entre 0 y 2 errores.

\section{DICTADO DE VOCALES, CONSONANTES Y NÚMEROS}

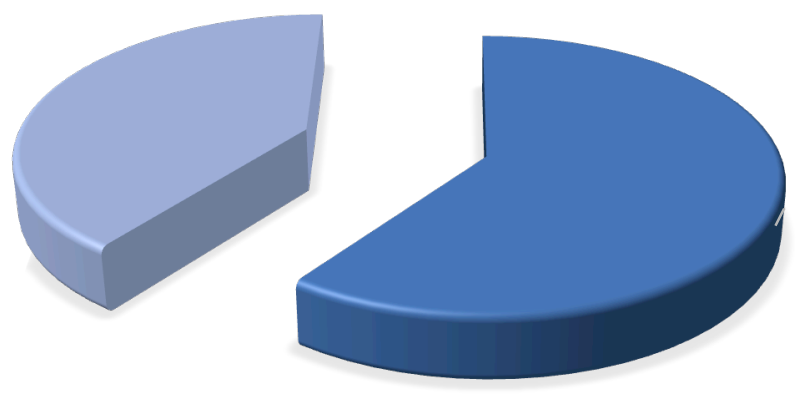

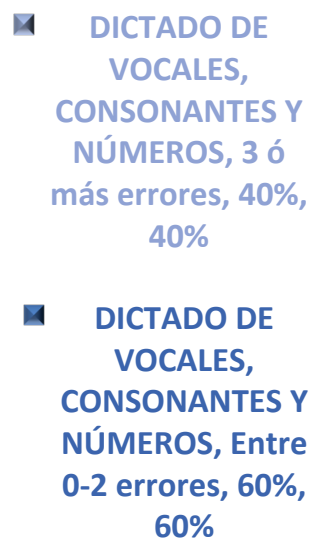

Figura 1: Prueba de lectoescritura

Si realizamos un corte transversal podemos observar respecto a la valoración de las vocales que en la letra A hay 3 alumnos que no la escriben correctamente; la letra E la escribe correctamente 1 alumno; la letra I la escriben correctamente 2 alumnos; mientras que las letras $\mathrm{O}$ y $\mathrm{U}$ la escriben correctamente todos. Los porcentajes del alumnado que comete errores quedarían reflejados en el siguiente gráfico.

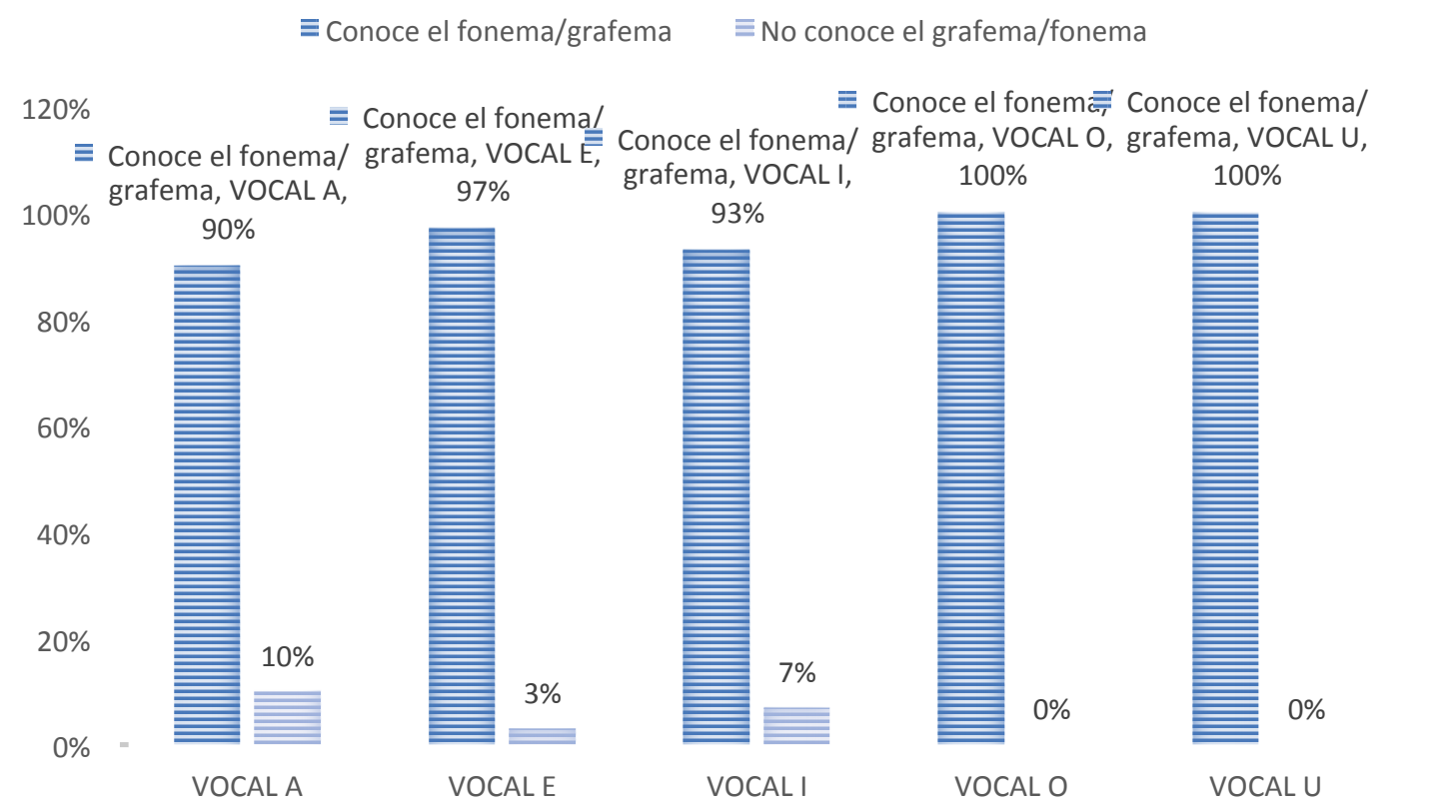

Figura 2: Alumnado que conocen las vocales

En la prueba en la que se analizaban los patrones básicos de movimiento las puntuaciones más bajas se sitúan tanto en arrastre como en triscado y carrera en 4 puntos, en control postural en 3 puntos y en tono muscular en 2. 


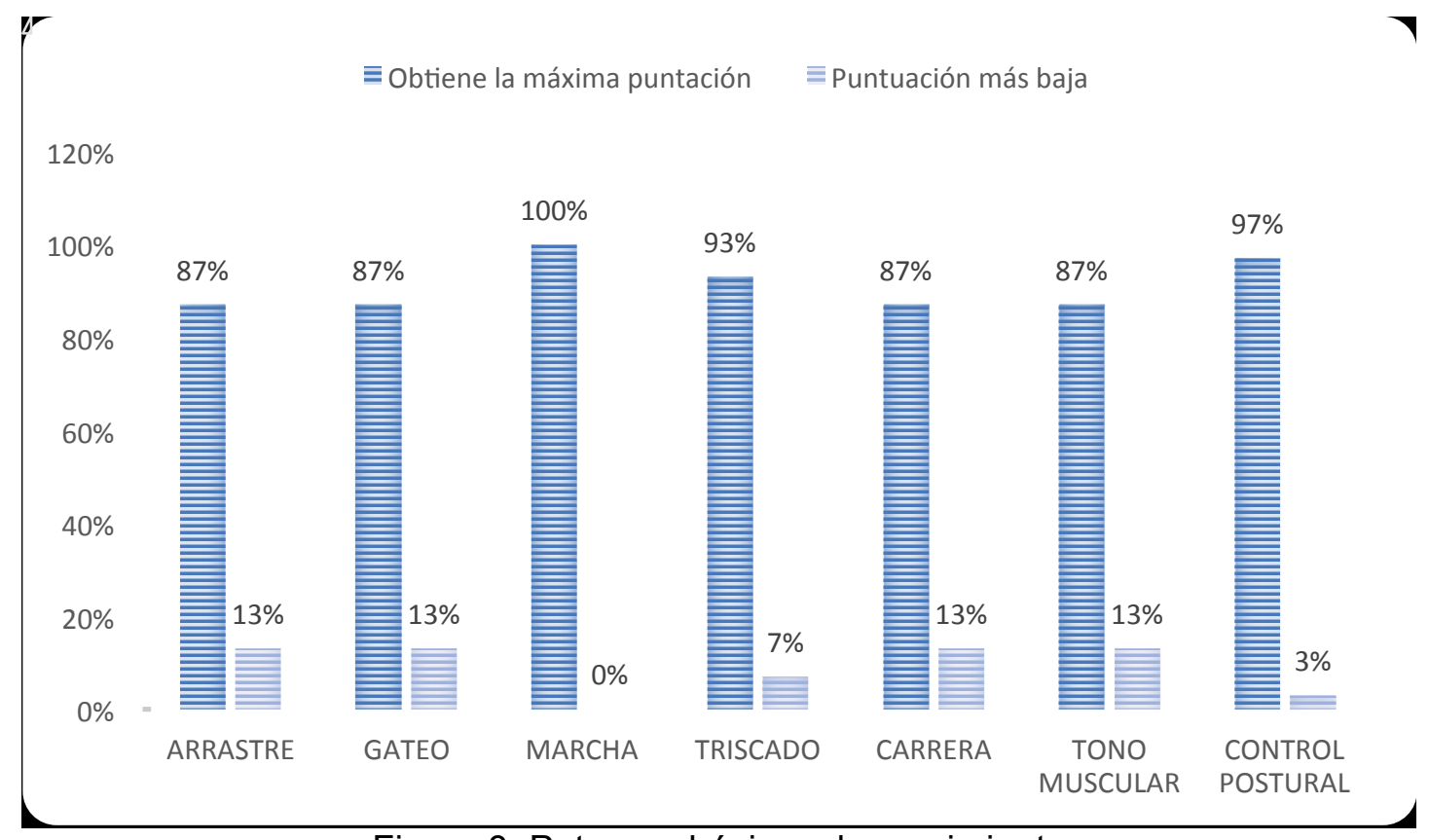

Figura 3: Patrones básicos de movimiento

En definitiva, podemos decir que hay 11 alumnos con puntuaciones bajas y 19 alumnos con puntuaciones altas, teniendo en cuenta que la máxima puntuación puede ser de 5 puntos.

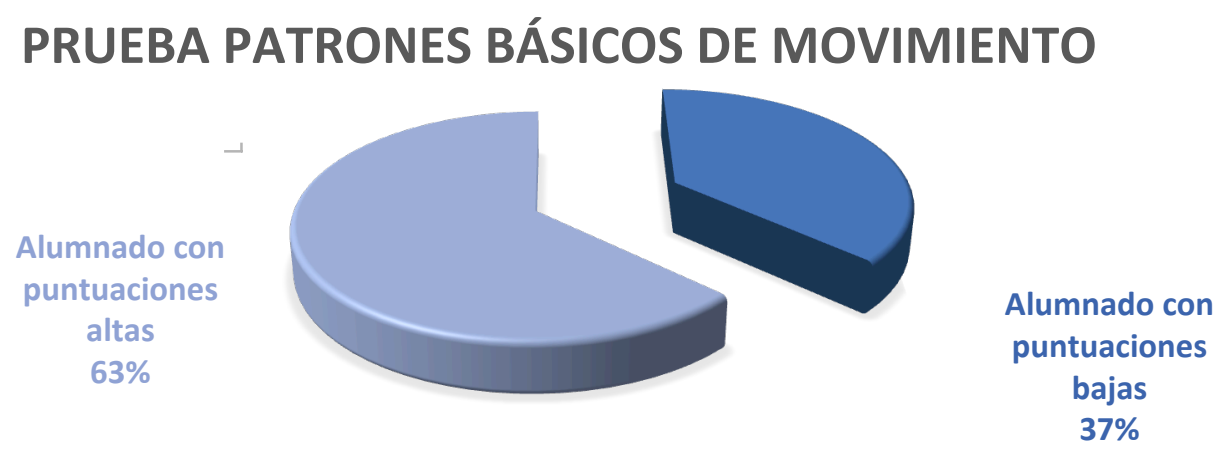

Figura 4: Patrones básicos de movimiento

Por todo ello podemos afirmar que un $63 \%$ de la muestra manifiesta un adecuado desarrollo psicomotor.

En la prueba de lateralidad hemos obtenidos resultados muy interesantes: contamos con 18 alumnos diestros, 1 zurdo, 10 con lateralidad cruzada y 1 sin evaluar. El alumno que está sin evaluar es porque sus características individúales no han permitido completar las pruebas que se pretendían pasar, por lo que no ha podido realizarse una evaluación completa de todos los ítems. 


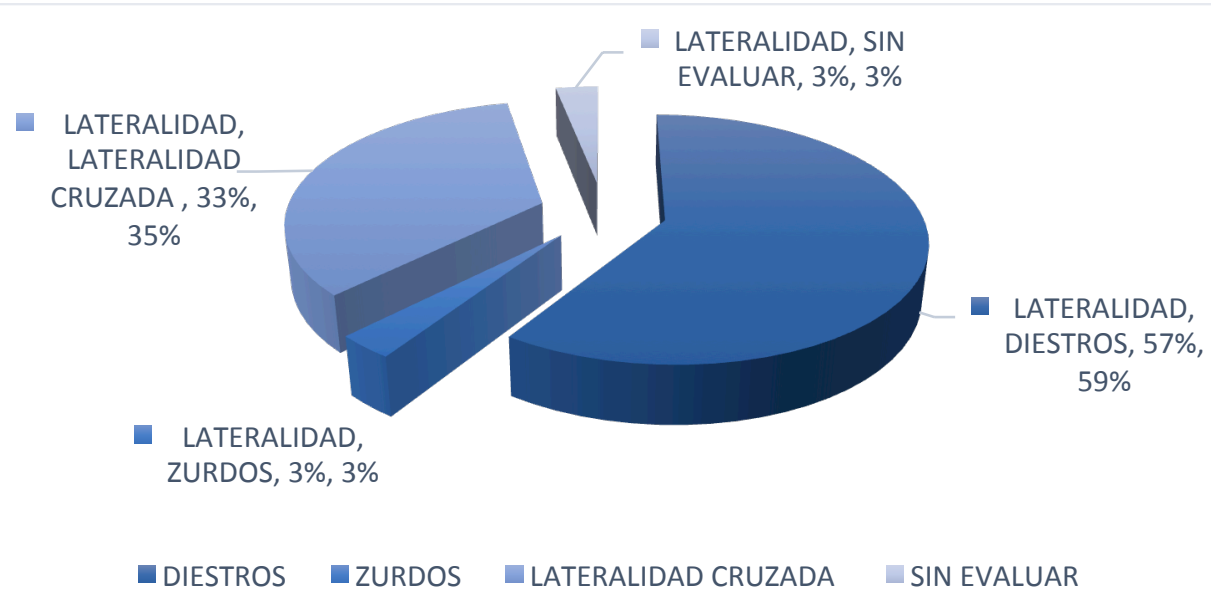

Figura 5: Lateralidad

A continuación se muestra una gráfica en la que aparecen el número de errores que han cometido en las pruebas de lectoescritura.

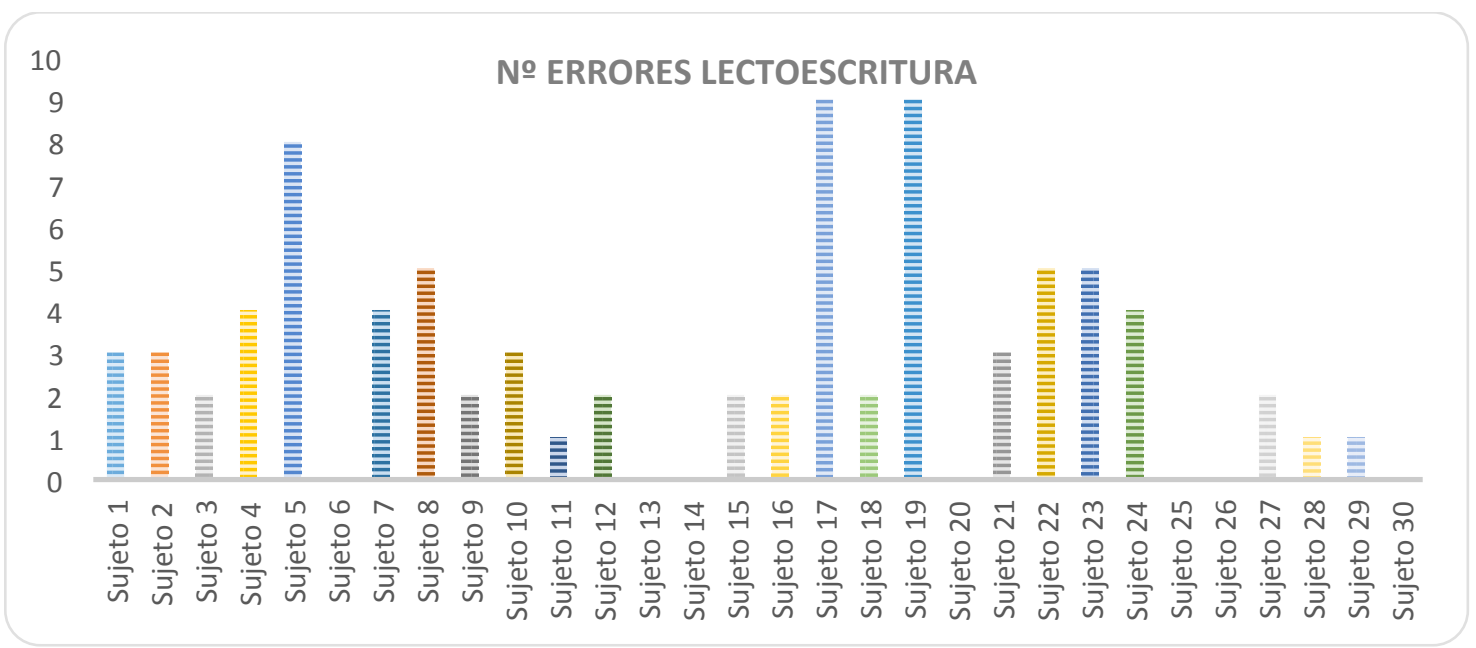

Figura 6: $\mathrm{N}^{\circ}$ de errores lectoescritura

Llegados a este punto, es fundamental conocer la relación entre los alumnos que han cometido un mayor número de errores en la prueba de lectoescritura con los que han obtenido puntuaciones más bajas en la prueba de patrones básicos de movimiento.

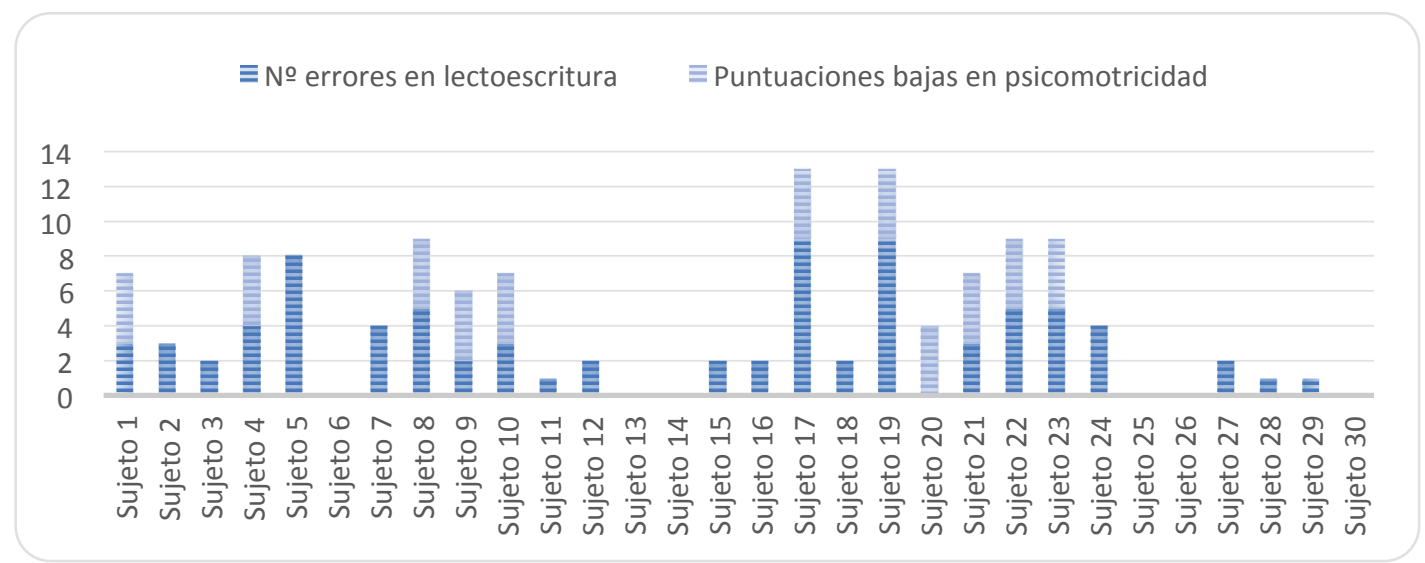

Figura 7: Puntuaciones en psicomotricidad y lectoescritura 
La interpretación del gráfico refleja que un total de 9 alumnos (sujetos 1, 4, 6, 10, 17 , $19,21,22$ y 23) cometen un mayor número de errores en la prueba de lectoescritura y obtienen puntuaciones más bajas en la prueba de patrones básicos de movimiento.

Sin embargo, hay 4 alumnos (sujetos 2, 5, 7 y 24) que cometen un mayor número de errores en la prueba de lectoescritura y no obtienen puntuaciones más bajas en la prueba de patrones básicos de movimiento, aunque hemos de tener en cuenta que el número de errores de estos alumnos se sitúan en $3,8,4$ y 4 , de los cuales, el segundo y la última tienen lateralidad cruzada según las pruebas realizadas de psicomotricidad. Por otra parte, podemos observar que hay 15 alumnos con un número de errores bajo en lectoescritura y una alta puntuación en la prueba de patrones básicos de movimiento.

Además, hemos de mencionar a 2 alumnos (sujeto 9 y 20) que tienen un bajo número de errores en lectoescritura y han obtenido una puntuación baja en la prueba de patrones básicos de movimiento, en concreto, tono muscular.

\section{CORRELACIÓN PRUEBAS DE LECTOESCRITURA Y PSICOMOTRICIDAD}

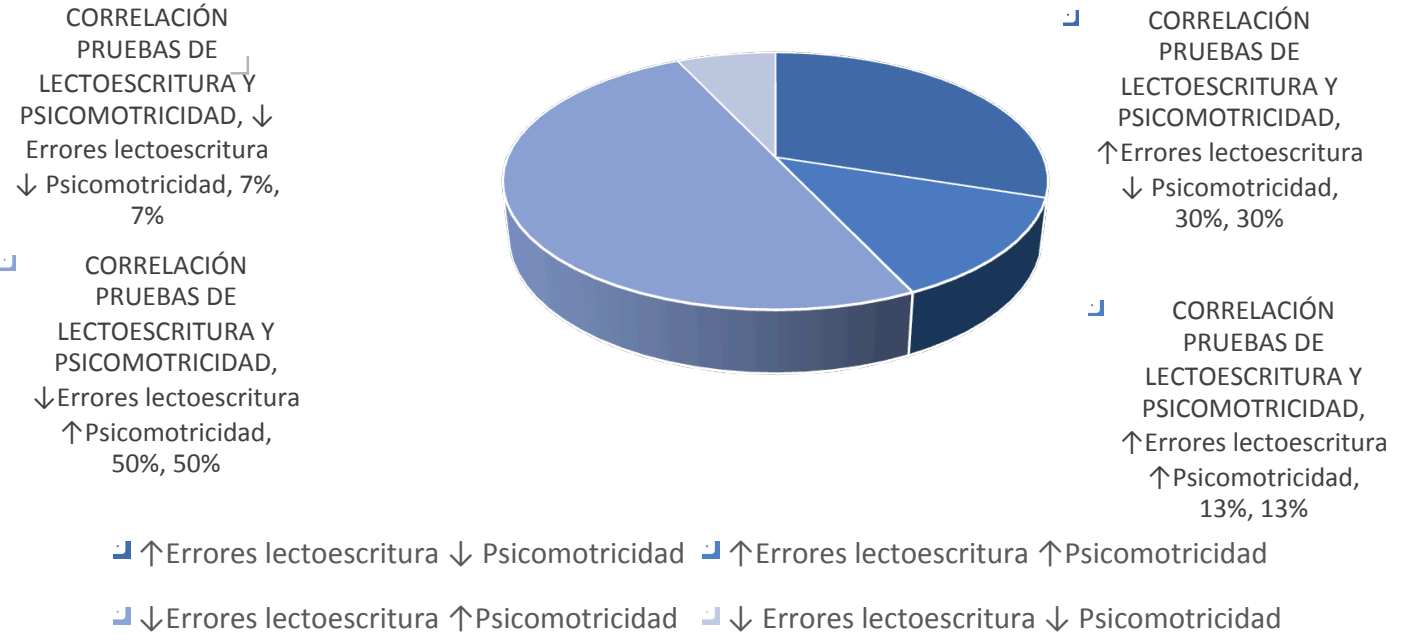

Figura 8: Puntuaciones en psicomotricidad y lectoescritura

\section{Discusión}

Los resultados que hemos obtenido a rasgos generales con el presente estudio confirman la importancia que recae en la psicomotricidad en el periodo de Educación Infantil. Hemos de volver a mencionar que los alumnos que han obtenido buenas puntuaciones en la prueba de lectoescritora lo representan un $60 \%$. En el caso de buenas puntuaciones en psicomotricidad, podemos hacer referencia a un $63 \%$ del alumnado, mientras que en lateralidad contamos con 18 alumnos diestros, 1 zurdo, 10 con lateralidad cruzada y 1 sin evaluar. Pero realmente, en el caso que estamos abordando, lo que nos interesa saber es hasta que punto correlacionan los alumnos que han cometido un mayor número de errores en la prueba de lectoescritura con los que han obtenido puntuaciones más bajas en la prueba de patrones básicos de movimiento. 
Dicho de otro modo, la pregunta que cabe esperar que nos hagamos si cruzamos los resultados obtenidos es si existe una correlación entre el alumnado que tiene un buen desarrollo psicomotor y buenos conocimientos en lectoescritura y viceversa.

La evidencia que nos ha aportado este estudio es que efectivamente un $50 \%$ de la muestra tiene buen desarrollo lectoescritor y psicomotor, un 30\% manifiesta un bajo desarrollo psicomotor y lectoescritor. Del $20 \%$ restante, nos encontramos que un $13 \%$ (4 alumnos) cometen un mayor número de errores en la prueba de lectoescritura y no obtienen puntuaciones más bajas en la prueba de patrones básicos de movimiento. Sin embargo, hemos de tener en cuenta que dos de ellos tienen lateralidad cruzada según las pruebas realizadas de psicomotricidad. El $7 \%$ restante (2 alumnos) muestran un bajo número de errores en lectoescritura y han obtenido una puntuación baja la prueba de patrones básicos de movimiento, en concreto, tono muscular. En conjunto, estos resultados nos sugieren que existe correlación entre un adecuado desarrollo psicomotor y un correcto aprendizaje de procesos lectoescritores.

Por otra parte, hemos de indicar que hemos alcanzado los objetivos propuestos en los inicios del presente estudio pues el objetivo general era trabajar la psicomotricidad desde el plano neuropsicológico en la etapa de Educación Infantil, concretamente en alumnos de 5 años, Se demuestra la relación existente entre psicomotricidad y procesos lectoescritores.

\section{Referencias}

Aucouturier, Lapierre (2000). El cuerpo y el inconsciente en educación y terapia. Editorial Científico Médica.

Chaddock-Heyman, L., Erickson, KI., Holtrop, JL., Voss, MW., Pontifex, MB., Raine, LB., Hillman, CH., \& Kramer, AF. (2014). Aerobic fitness is associated with greater white matter integrity in children. Front. Hum. Neurosci. 8, 584. doi: 10.3389/fnhum.2014.00584.

De Lièvre, B., \& Staes, L. (1992): La psychomotricité au service de l'enfant. Paris: Belin.

Hannaford, C. (1995). Como aprende tu cerebro. México: Editorial Pax México.

Henrietta C. Leiner, Alan L. Leiner, Robert S. Dow. (1993).Cognitive and language functions of the human cerebellum. Trends in Neurosciences, 16(11), 444-447.

Martín Lobo, P. (2003). La lectura. Procesos neuropsicológicos del aprendizaje, dificultades, programas de intervención y estudio de casos. Barcelona: Lebón.

Martín Lobo, P., García Castellón, C., Rodríguez, I. y Vallejo, C. (2011). Test de lateralidad de la prueba neuropsicológica. Instituto de Neuropsicología y Educación. Madrid: Fomento.

Satiuste, V., Martín Lobo, M.P y Ayala, C. (2005). Bases neuropsicológicas del fracaso escolar. Madrid: Fugaz.

Strick, PL. (2009). Cerebellum and Nonmotor Function. Annual Review of Neuroscience, 32, 413-434. 